\title{
Control of responding by a percentage reinforcement schedule'
}

SHIN-HO CHUNG AND ALLEN J. NEURINGER

FOUNDATION FOR RESEARCH ON THE NERVOUS SYSTEM

When a fixed-interval schedule requirement is sometimes followed by food reinforcement and sometimes by a neutral stimulus, this stimulus comes to approximate the reinforcing functions of food.

A previously neutral stimulus, if presented at the same time as, or immediately preceding, a primary reinforcer, acquires the property of a secondary reinforcer and exerts control over operant behavior. It was recently demonstrated that a neutral stimulus which intermittently replaces the primary reinforcer also exerts considerable control over responding (Neuringer \& Chung, 1967). When a small fixed-interval component was sometimes followed by food and sometimes by a neutral stimulus, pigeons responded as if food followed every component. On this schedule, designated as a percentage reinforcement, or PR, schedule, primary reinforcement and neutral stimulus were never temporally contiguous. The neutral stimulus, however, exerted reinforcing control. The present study attempts to determine the extent to which such a stimulus approximates the function of food. To do this, the behavior obtained under a regular fixedinterval schedule-all intervals terminating with foodis parametrically compared to the behavior under a comparable percentage reinforcement schedule. Subjects

Forty male White Carneaux pigeons, 11 months old at the beginning of the experiment, were maintained at approximately $90 \%$ of their free-feeding body weights. Apparatus

Three standard experimental chambers for pigeons, as described by Ferster \& Skinner (1957), were used. Each chamber contained a response key which was transilluminated by a 7-W bulb. Located directly below the response key was a feeder which gave 3-sec. access to food for reinforced responses. A peck of at least $10 \mathrm{gm}$ was recorded and produced auditory feedback for the pigeon. All sessions terminated after 30 reinforcements.

Precedure

$100 \%$ condition. The basic schedule of reinforcement under this condition was a response-initiated fixedinterval schedule (Mechner et al, 1963). A peck initiated a fixed interval (FI), and the first peck to occur after the interval produced a food reinforcement. Since all intervals terminated with food, this will be referred to as the $100 \%$ condition. The duration of the FI components was the main independent variable.

The Ss were randomly divided into 10 groups of four. Each group was placed under one of the following
FI schedules: $1,2,3,4,5,6,8,10,12$, and 15 sec. After 33 sessions, the Ss were re-divided into 10 different groups randomly, with the provision that no $S$ would be assigned to the same FI schedule to which it was assigned previously. After another 33 sessions, the same re-division occurred and Ss received another 32 sessions. Eight pigeons, four after the end of the first 33 sessions and the other four after the end of 66 sessions, were sacrificed for a neurochemical study.

Percentage reinforcement (PR) condition. The basic schedule under this condition was, again, a responseinitlated FI schedule. Under this schedule, however, food followed only some of the FI components. All other components terminated with a 1-sec. blackout period during which the experimental chamber was totally dark. During blackouts, pecks did not produce feedback clicks. It was found that virtually no responses were emitted in these blackout periods. Food reinforcements were scheduled by a variable interval (VI) program with an average inter-reinforcement interval of $1 \mathrm{~min}$. The VI programmer was activated at all times throughout the session except when it had programmed a reinforcement and during periods of blackout and reinforcement. If the VI programmer had primed, a FI component would end with a response-produced reinforcement. If the VI programmer had not primed, the same component would end with a responseproduced blackout. As in the $100 \%$ condition, the duration of the FI component was the main independent variable.

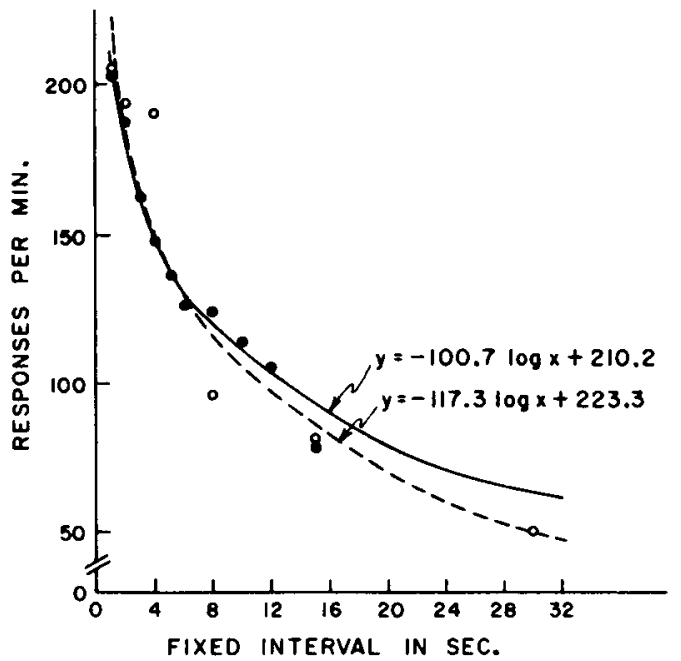

Fig. 1. Rate of responding as a function of fixed interval for $100 \%$ (closed circles and solid line) and percentage reinforcement (open circles and broken line) conditions. 


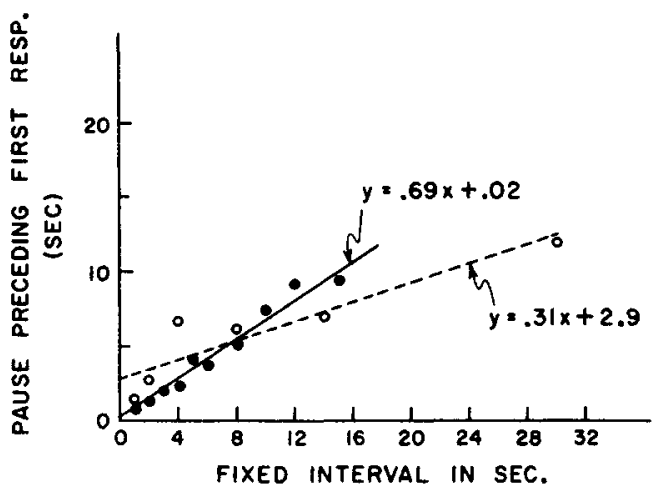

Fig. 2. Pause preceding each FI component as a function of the fixed interval for $100 \%$ (closed circles and solid line) and percentage reinforcement (open circles and broken line) conditions.

Thirty of the Ss used in the previous condition were randomly selected and assigned to six groups of five pigeons each. Each group was placed on a percentage reinforcement schedule consisting of one of the following fixed intervals: $1,2,4,8,15$, and 30 sec. Although the FIs differed, the frequency of food was the same for all groups, i.e., one reinforcement/ min. Each $\mathrm{S}$ was given 35 sessions ${ }^{\circledR}$ exposure on the schedule.

\section{Resulfs}

Under both PR and $100 \%$ conditions, each fixed interval was initiated by a response. Thus, the behavior under both conditions can be divided into (a) the average pause preceding the first response, and (b) the average rate of responding during the FI component. In Fig. 1, average rate of responding after pause is plotted as a function of fixed interval for both $100 \%$ (filled circles) and PR (open circles) conditions. Each of the points in Figs. 1 and 2 is a geometric average of the final three sessions' performances. The logarithmic functions in Fig. 1 minimize the sum of the squared deviations between predicted and observed values. With increasing FT, the rates of responding under both conditions decreased rapidly and reached an asymptote. It is noteworthy that the parameters of the two functions are remarkably similar.

Figure 2 shows average pause as a function of fixed interval for both $100 \%$ (filled circles) and PR (open circles) conditions. The linear functions relating pause to fixed interval were again estimated according to least squares analyses. The points obtained from the PR group are not as orderly as those obtained from the $100 \%$ group. This might be due to the different sample sizes used in the two conditions. Discussion

The central fact disclosed by the present study is that, under both percentage reinforcement and regular fixed-interval conditions, pause increased linearly and response rate decreased logarithmically with increasing FI. The parameters of the corresponding functions did not differ appreciably. These findings suggest that the blackout stimulus, when presented according to the PR schedule, approximated the function of a food reinforcer.

Since all Ss were first exposed to the $100 \%$ and then to the PR condition, it might be argued that the PR effects obtained here were due to "transfer." However, similar PR effects have been obtained from Ss having no prior training under a $100 \%$ condition (Neuringer \& Chung, 1967). Thus, the reinforcing effects of the blackout stimulus appear to be a function of the PR schedule.

The fixed-interval contingencies in the present study exerted relatively greater control over responding than did the frequency, or probability, of food per se. Whereas the frequency of food was always less under the PR condition than under the $100 \%$ condition, the fixed-interval contingencies were, at each comparison, identical. As was shown, responding under the two conditions was similar.

\section{References}

Ferster, C. B., \& Skinner, B. F. Schedules of reinforcement. New York: Appleton-Century-Crofts, 1957.

Mechner, F., Guevrekian, L., \& Mechner, V. A fixed interval schedule in which the interval is initiated by a response. $J$. exp. Anal. Behav., 1963, 6, 323-330.

Neuringer, A. J., \& Chung, S. H. Quasi-reinforcement: control of responding by a percentage reinforcement schedule. J. exp. Anal. Behav., 1967, 10, 45-54.

\section{Note}

1. This work was supported by a grant (MH 12108-01) from the National Institutes of Health. Reprints may be obtained from Allen J. Neuringer, Foundation for Research on the Nervous System, 36 The Fenway, Boston, Massachusetts 02215. 